\title{
Bootstrap Approximation of Gibbs Measure for Finite-Range Potential in Image Analysis
}

\author{
Abdeslam EL MOUDDEN \\ Business and Management School \\ Ibn Tofaïl University \\ Kenitra, Morocco
}

\begin{abstract}
This paper presents a Gibbs measure approximation method through the adjustment of the associated estimated potential. We use the information criterion to prove the accuracy of this approach and the bootstrap computation method to determine the explicit form. The Gibbs sampler is the tool of our simulations while taking advantage of the use of the only one MCMC inside of the multiple necessary MCMC in the classical approximation. We focus on the validity of our approach for the Gibbs measure of a Markov Random Field with an interaction potential function and the associated uniqueness condition. Some theoretical and numerical results are given.
\end{abstract}

Keywords-bootstrap computation; Gibbs measure; Markov Chain Monte Carlo; image Analysis; parameter estimation. Likelihood inference

\section{INTRODUCTION}

It is well known that in computer vision, one of the first aims is to determine, for generic prior Gibbs model, the correct form of the potential function. Certainly, this later will be better if the parametric estimation is good enough [5], but this requires both ideal conditions and expensive costs. Furthermore, the Gibbs Random Fields have become an efficient instrument in image analysis. So, the associated statistical inference has attracted a great deal of interest, because of its great adequacy in important applications related to image processing, computer vision, neural modeling and perceptual inference. Nevertheless, to estimate the true parameter for the Gibbs model required a high cost in terms of computation time and modeling conditions. For example, the use of the maximum likelihood estimation (MLE) was impossible to be calculated and was substituted by the pseudolikelihood estimation [5] [18]. Fortunately, the intense developments in statistics accompanied by the evolution in computer systems allowed maximum likelihood estimation for Gibbs Random Fields to be constructed. For this, we propose the use of the resampling method [3] through Markov Chain Monte Carlo (MCMC). The bootstrap computation method and MCMC with Gibbs sampler is used to retrieve ever more the desired potential function for the parameter Gibbs distribution.

Some technical changes make use of one MCMC instead of two chains, which is very promising to reduce the computational time. We prove that the KL-distance is minimized for the adjusted potential function. Moreover, the adjustment method proposed in this paper keeps the features of prior potential function for the associated Gibbs measure.
This paper is organized as follows. In section 2, we present the necessary context of the Gibbs models that describes the validity of the proposed approximation. In section 3 , we present the steps of the approximation proposed in this paper in particular the study of the information criterion for a Gibbs model from what we inspire the proper form of the adjusted potential and prove the accuracy of the associated approximation even though by the use of one MCMC . In section 4, we present some numerical results to explain the feasibility of the usefulness of the approximated expressions.

\section{THE THEORITICAL APPROACH OF A GIBBS RANDOM FIELD WITH POTENTIAL INTERACTION FUNCTION}

Before presenting the adjustment method for a parametric potential interaction function of a Gibbs distribution, it is necessary to review the concepts and results related to the Gibbs measure, which will clarify the necessity and importance of using the bootstrap approach and techniques that we introduce in this context.

\section{A. Click and neighborhood system}

It is quite obvious that a digital image is modeled by a matrix $X$ of data on a network $S \subseteq \mathbb{Z}^{2}$, instead of a linear data base. The shape of such model is a Random Field $X=\left(X_{s}, s \in S\right)$ instead of an ARMA model for example.

In the network $S$, a system of neighborhood $\mathcal{V}=$ $\left\{V_{s} ; s \in S\right\}$ is defined as follows:

- $s \notin V_{s} ;$ for $s \in S$

- for $s, t \in S ; s \in V_{t} \Leftrightarrow t \in V_{s}$

$V_{s}$ is a set of neighboring elements of $s$. So, a part $C$ of $S$ is called a click with respect to $\mathcal{V}$ if:

- $C$ is reduced to a single site

- or it contains at least two elements and each pair (s, t) of elements is formed of neighboring sites (with regard to $v)$.

The boundary of a non-empty subset $V$ of the network $\mathrm{S}$ is a subset $\partial \mathrm{V}$ defined as:

for $s \in \partial V$ there exists an element $t \in V$ such that, $s \in V_{t}$

Given a distance $d$ on $\mathrm{S}$, the neighborhood $V_{s}$ of a site $S$ with respect to a finite-range $\ell$ is:

$$
\begin{gathered}
V_{s}=\{t \in S-\{t\}: d(s ; t) \leq \ell\} \\
\text { So, the boundary of a subset } V \text { of length } \ell \text { is, } \\
\partial V_{\ell}=\{s \in S: \exists t \in V \text { such that } d(s ; t) \leq \ell\}
\end{gathered}
$$




\section{B. Random field on a nethwork}

We consider a random field $X=\left(X_{s}, s \in S\right)$ defined on a network $S \subseteq \mathbb{Z}^{2} . X$ takes its values in the set of configurations

$$
\Omega=\Omega(\mathrm{S})=E^{S}
$$

$E$ is the set of the different levels of a pixel s. The first way to define a probability measure of the random field $X$ on $\Omega$ is to give a Kolmogorov projective family of marginal distribution on finite subsets $\mathrm{V}$ of $\mathrm{S}$. Nevertheless, the right way is to define a kernel family of conditional probabilities [11], which is more appropriate in case of image processing and analysis. In other words, given a probability measure $v$ on $\Omega$, the conditional probability kernel on a subset $\mathrm{V}$ of $\mathrm{S}$ is defined as follows:

with,

$$
\begin{array}{r}
P^{V}(., .): \Omega(S-V) \times \mathfrak{I}(V) \rightarrow \quad[0,1] \\
(y, A) \rightarrow P^{V}(y, A)
\end{array}
$$

$$
P^{V}(y, A)=E_{v}\left(1_{A} / y\right)
$$

is the conditional expectation on the Borel space $\mathfrak{I}(V)$ given a configuration $y$ on the outside $(S-V)$ of $\mathrm{V}$, such that for all $V^{\prime} \subset V$ and $A \in \mathfrak{I}(S)$, we have almost surely: $E_{v}\left[E_{v}\left(1_{A} / \mathfrak{I}(S-V)\right) / \mathfrak{I}\left(S-V^{\prime}\right)\right]=E_{v}\left(1_{A} / \mathfrak{I}\left(S-V^{\prime}\right)\right)$

This can be written as the following:

$$
\int P^{V}(x, A) P^{V^{\prime}}(y, d x)=P^{V^{\prime}}(y, A)
$$

$v$-almost surely for all $y \in \Omega\left(S-V^{\prime}\right)$. It is expressed as an operation " $\star$ " between two kernels $P$ and :

$$
P \star Q(z, A)=\int Q(x, A) P(z, d x)
$$

Using the operation " $\star$ " above, we give the definition of a specification below. $f p(S)$ denotes the set of finite subsets of the network $\mathrm{S}$.

\section{Definition 1.1}

A specification is a family $\left(\pi^{V}\right)_{V \in f p(s)}$ of probability kernels satisfying:

a) For $V \in f p(S)$ and $A \in \mathfrak{I}(S), \pi^{V}(., A)$ is a random variable $\mathfrak{I}(S-V)$-measurable.

b) For all $V \in f p(S), \pi^{V}(., A) \equiv 1_{A}$ if $A \in(S-V)$.

c) For all $V$ and $V^{\prime} \in f p(S), \pi^{V^{\prime}} \star \pi^{V} \equiv \pi^{V^{\prime}}$.

Then, it follows that for a Markov Random Field given a Gibbs measure $v$, the associated specification can be written as (Dobrushin-Lanford-Ruelle-equation):

for $V \in f p(S)$,

$$
\pi^{V}(., .)=E_{v}(. / \Im(S-V))
$$

The sufficient condition of the existence of a Gibbs measure given a specification can be written as:

$$
\forall V \in f p(S) \text { and } A \in \mathfrak{I}(V) \text {, }
$$

$$
\sup _{y \in \Omega}\left|\pi^{V}(y(S-V), A)-\pi^{V}\left(y\left(\partial_{\ell} V\right), A\right)\right| \underset{\ell \rightarrow+\infty}{\longrightarrow} 0
$$

$y(S-V)$ and $y\left(\partial_{\ell} V\right)$ are the restriction configurations to $(S-V)$ and $\partial_{\ell} V$ respectively. It should be noted that the condition (7) is true for a Markov Random Field with finiterange potential.

\section{The interaction potential function of a GibbsRandom field}

For a Markov Random field, the associated Gibbs measure " $v$ " is defined via the specification like:

$$
\pi^{D}(y, A)=\int_{A} \frac{1}{z_{D}(y)} \exp \left[-H_{D}(x . y)\right] d v(x)
$$

for all $D \in f p(S)$ and $y \in \Omega(S-D)$. Then " $v$ " is a positive measure defined on $\Omega$ and $H_{V}(.,$.$) is the energy function$ given by the potential $\left(I_{V}\right)_{V \in f p(S)}$ such that, for $x \in \Omega(D)$ and

$y \in \Omega(S-D)$ :

$$
H_{D}(x \cdot y)=\sum_{V, V \cap D \neq \Phi} I_{V}(x \cdot y)
$$

$(x . y)$ is the concatenation of the configuration $x$ on $\mathrm{D}$ with boundary condition $y$ on (S-D). We can recall the different results in this context; however, we are interested in conditions of existence and uniqueness of the Gibbs measure given a potential function specification. It is used in measuring the accuracy of parametric model estimation for a Markov random field. Because of this, it is introducing a quantity, for a given site $s$ on $\mathrm{S}$ private the origin $\mathrm{o}$ :

$$
\rho_{s}=\sup \frac{1}{2}\left\|\pi^{o}(y, .)-\pi^{s}\left(y^{\prime}, .\right)\right\|
$$

The sup is taken over all configurations $y$ and $y^{\prime}$ on $S-\{o\}$ identical everywhere except on s, and $\|$. $\|$ denotes the total variation norm of a measure $\mu$, defined by:

$$
\|\mu\|=\sup \left\{|\mu(f)| ;\|f\|_{\infty}=1\right\}
$$

The quantity $\rho_{s}$ measures the maximum influence of the modality at the site $s$ on the conditional distribution at the origin network of S. So, the uniqueness condition of a Gibbs measure [11] is of the form:

$$
\sum_{s \neq o} \rho_{s}<1
$$

This is rewritten in [7] for a specification with a potential invariant under translation as well; $\exists \alpha \in[0,1$ [ such that,

$$
\forall s \in S, \quad \sum_{V \in f p(s), V \ni s}(|V|-1)\left\|I_{V}\right\|_{\infty}<\alpha
$$

The expression (12) is more significant and useful than (11).

\section{Markov Chain Monte Carlo and paremeter estimation for Gibbs distribution}

We can find some papers that dealt with the maximum likelihood estimation for a Gibbs Random Field [5] [19]. The main problem in this topic is essentially the large computation time for a complete determination of the MLE based on maximizing:

$$
\pi_{\theta}^{D}\left(x_{o} / y\right)=\exp \left(-H_{D}\left(\theta, x_{o} \cdot y\right)\right) / z(\theta)
$$

for an observed configuration $x_{o}$ of the Gibbs Random Field $X$ on a subset $D \in f p(S)$, where $y$ is a boundary configuration outside $D$. the expression (13) is the parametric version of (8) with respect to the parametric potential $\left(I_{V}(\theta, .)\right)_{V \in f p(s)}$. The normalization constant is:

$$
z(\theta)=\sum_{x \in \Omega(D)} \exp \left(-H_{D}(\theta, x, y)\right)
$$

Recently the construction of the MLE is allowed due to computational system evolution. It is to solve the derivative equation:

$$
E_{\theta}\left(\nabla H_{D}(\theta, X)-\nabla H_{D}\left(\theta, x_{o}\right)=0\right.
$$


$\nabla$ represents the gradient operator of partial derivative with respect to the parameter coordinates $\theta \in \Theta \subseteq \mathbb{R}^{p}$ where $p \geq 1$. The solution $\widehat{\theta}$ (i.e. the MLE) of (14) in $\theta$ is approximated by the limit of a stochastic sequence through a dynamic markovian algorithm using a $\operatorname{MCMC}\left(X^{j, n}\right)_{1 \leq j \leq N}$ via a Gibbs sampler controlled by the $\theta_{n}$ term of the sequence:

$$
\theta_{n+1}=\theta_{n}+\alpha\left(\widehat{E}_{\theta_{n}}\left(\nabla H_{D}\left(\theta_{n}, X\right)-\nabla H_{D}\left(\theta_{n}, x_{o}\right)\right)\right.
$$

where,

$$
\widehat{E}_{\theta_{n}}\left(\nabla H_{D}\left(\theta_{n}, X\right)=\sum_{j=1}^{N} \nabla H_{D}\left(\theta_{n}, X^{j, n}\right) / N\right.
$$

And it is allowed under the Gibbs sampler ergodic property.

\section{ADJUSTMENT OF THE INTERACTION POTENTIEL FUNCTIION OF A GIBBS MEASURE}

In this section, we to introduce the bootstrap methodology [4], as an adjustment tool for the parametric energy function $H_{D}(\hat{\theta}, x, y)$, given in (13), to obtain a more appropriate Gibbs measure compared to that associated with the estimated potential. This is proved using the information criterion.

\section{A. Information Criterion and Variational Principle of Gibbs Random fields}

In the framework of the modeling of a random field on a regular network, it is proved that the variational principle [21], i.e. the decision to assign a Gibbs measure " $v$ " of a random field $X$ when the true one is " $\mu$ ", can be expressed in terms of an information criterion $h(v, \mu)$, and this vanishes if and only if $\mu=v$. The same result is given [8] in the restricted case; the information criterion $h(v, \mu)$ vanishes if and only if $\mu$ and $v$ have the same interaction potential.

For this reason it is obvious that the true Gibbs measure is not necessarily to be associated with the estimated potential $\left(I_{V}(\hat{\theta}, .)\right)_{V \in f p(S)}$; the fact that the maximum likelihood estimator for the Gibbs fields is substantially biased [19], which is the same case for the pseudo likelihood estimator [5]. So, the following adjustment of the estimated potential proposed in this paper has a considerable magnitude to dig up the accurate specification of the parametric Gibbs measure.

Doing this, we consider firstly the kullback-Leibler function applied to the parametric Gibbs measure $P_{\theta}$ and to another any Gibbs measure $Q$,

Where,

$$
K\left(P_{\theta}, Q\right)=\lim _{D} \inf K_{D}\left(P_{\theta}, Q\right)
$$

$$
K_{D}\left(P_{\theta}, Q\right)=\left\{\begin{array}{cc}
E_{\theta}\left[\log \left[\frac{d P_{\theta, D}}{d Q_{D}}\right]\right], & \text { if } P_{\theta, D} \ll Q_{D} \\
\infty, & \text { otherwis }
\end{array}\right.
$$

The symbol " $\ll$ " means that $P_{\theta}$ is absolutely continuous with respect to $Q_{D} \cdot \frac{d P_{\theta, D}}{d Q_{D}}$ is the Radon-Nikodym derivative of the two restricted measures on the finite subset $D$ of the lattice $S$. The specific information of $P_{\theta}$ with respect to $Q$ is:

$$
h\left(P_{\theta}, Q\right)=\lim _{D} \inf \frac{1}{|D|} K_{D}\left(P_{\theta}, Q\right)
$$

We recall that if $P_{\theta}$ is the Gibbs measure of the specification $\left(\pi^{V}\right)_{V \in f p(S)}$ then, $h\left(P_{\theta}, Q\right)=0$ if and only if $Q$ has the same specification $\left(\pi^{V}\right)_{V \in f p(s)}$. It means that for a specification with a finite-range interaction potential, $h\left(P_{\theta}, Q\right)=0$ if and only if $P_{\theta}$ and $Q$ have the same interaction potential.

\section{B. Adjustement of the Gibbs measure potential}

For a parametric Gibbs model, we note the following condition:

$$
\theta_{1} \neq \theta_{2} \Rightarrow G\left(\theta_{1}\right) \cap G\left(\theta_{2}\right)=\Phi
$$

It means that two different values of $\theta$ give two different Gibbs measures. So, under this condition [18] we have the following result:

$$
\operatorname{Lim}_{D>S} \frac{1}{|D|} \log \left[\frac{\pi_{\theta_{r}}^{D}(x)}{\pi_{\theta}^{D}(x)}\right]=-h\left(P_{\theta_{r}}, P_{\theta}\right)=-h\left(\theta_{r}, \theta\right)
$$

which exists almost surely, where $\theta_{r}$ is the true value of the parametric Gibbs model for a Markov Random Field X observed through an image $x$ on the finite subset $D$. So,

$$
h\left(\theta_{r}, \theta\right)=0 \Leftrightarrow \theta=\theta_{r}
$$

Furthermore, if $\Theta$ is a compact space of $\mathbb{R}^{p}$, there exists a constant $\beta \geq 0$, such that, for all $\theta_{1}$ and $\theta_{2}$ in $\Theta$ :

$$
\frac{1}{|D|}\left|\log \left[\pi_{\theta_{1}}^{D}(x)\right]-\log \left[\pi_{\theta_{2}}^{D}(x)\right]\right| \leq \beta \cdot\left|\theta_{1}-\theta_{2}\right|
$$

In practice, the observation window $\mathrm{D}$ is quite low with respect to the lattice $S$, in addition, the MLE has significant bias. So, the estimated value $\theta_{1}=\hat{\theta}$ is not so satisfying, but from (19) we can note for an estimation $\theta_{1}$ of $\theta_{r}$ :

$$
\log \left[\frac{\pi_{\theta_{r}}^{D}(x)}{\pi_{\theta_{1}}^{D}(x)}\right] \cong-|D| \cdot h_{D}\left(\theta_{r}, \theta_{1}\right)
$$

Also, from (9) and (13) we have:

$$
\pi_{\theta_{r}}^{D}(x) \cong \frac{\exp \left[-\sum_{V \cap D \neq \Phi} I_{V}\left(\theta_{1}, x\right)\right]}{Z_{D}\left(\theta_{1}\right)} \times \exp \left[-|D| \cdot h_{D}\left(\theta_{r}, \theta_{1}\right)\right]
$$

Then, this logical approximation induces the adjusted potential given an estimation $\theta_{1}$ of the unknown true value of the parameter Gibbs model as follows:

$\tilde{I}_{V}(x)=\left\{\begin{array}{cc}I_{V}\left(\theta_{1}, x\right)+\frac{|D|}{\left|\phi_{D}\right|} \cdot h_{D}\left(\theta_{r}, \theta_{1}\right), & \text { if } \operatorname{diam}(V) \leq \gamma \\ 0, & \text { otherwise }\end{array}\right.$

where $\gamma$ denote the finite-range of the potential $\left(I_{V}\right)_{V}$ and $\phi_{D}=\{V \in f p(S): V \cap D \neq \Phi$ and $\operatorname{diam}(V) \leq \gamma\}$.So, it is easy to verify that $\left(\breve{I}_{V}\right)_{V}$ is an interaction potential. This potential verifies the condition of Dobrushin's theorem for the uniqueness of Gibbs measure (12) as well as the estimated potential $\left(\mathrm{I}_{\mathrm{V}}\left(\theta_{1}, .\right)\right)_{\mathrm{V}}$. Indeed, from (21) and (24) we can write the following inequality for all $V \in f p(S)$ :

$$
\left\|\tilde{I}_{V}\right\|_{\infty} \leq\left\|I_{V}\left(\theta_{1}, .\right)\right\|_{\infty}+\frac{|D|}{\left|\phi_{D}\right|} \beta .\left\|\theta_{r}-\theta_{1}\right\|_{p},
$$

On the other hand, for a site $\mathrm{s} \in S$, we have:

$$
\sum_{V \ni S}(|V|-1)\left\|\tilde{I}_{V}\right\|_{\infty} \leq \alpha+\beta \cdot \sum_{V \ni S}(|V|-1) \frac{|D|}{\left|\phi_{D}\right|}\left\|\theta_{r}-\theta_{1}\right\|_{p}
$$

Also, 


$$
\sum_{V \ni S}(|V|-1)\left\|\tilde{I}_{V}\right\|_{\infty} \leq \alpha+\beta \cdot\left(\gamma^{2}-1\right) \frac{|D|}{\left|\phi_{D}\right|}\left|\phi_{\{s\}}\right| \cdot\left\|\theta_{r}-\theta_{1}\right\|_{p}
$$

It follows that the adjusted potential $\left(\check{\mathrm{I}}_{\mathrm{V}}\right)_{\mathrm{V}}$ satisfies uniqueness condition if:

$$
\left\|\theta_{r}-\theta_{1}\right\|_{p} \leq(1-\alpha) \frac{\left|\phi_{D}\right|}{|D| \cdot\left|\phi_{\{S\}}\right|}\left[\beta .\left(\gamma^{2}-1\right)\right]^{-1}=\varepsilon
$$

Note that for $\gamma=1$, the above result is trivial; otherwise, from the consistency of the Maximum Likelihood Estimator we can obtain for a sufficiently large window $D,\left\|\theta_{r}-\theta_{1}\right\|_{p} \leq$ $\varepsilon$.

\section{The Approximated Gibbs Measure}

We note by $\widetilde{P}$ the Gibbs measure associated to the adjusted potential $\left(\check{\mathrm{I}}_{\mathrm{V}}\right)_{\mathrm{V}}$. The initial parametric Markov random field $X$ has almost surly the same previous potential. In fact, the specification information in (17) is minimized by construction as in (22). Then, if we note $\left(\tilde{\pi}^{D}\right)_{D}$ the specification associated to $\left(\check{I}_{V}\right)_{V}$ in the sense of (8) and (9), we have exactly:

$$
\lim _{D} \inf h_{D}\left(P_{\theta_{r}}, \tilde{P}\right)=0
$$

What implies that, under the uniqueness condition, the two Gibbs measures $P_{\theta_{r}}$ and $\tilde{P}$ are the same. In the case of the nonuniqueness, $P_{\theta_{r}}$ and $\tilde{P}$ have at least the same interaction potential function.

\section{Bootstrap Approximation of Gibbs measure}

It is clear that the approximation Gibbs measure depends on the unknown value $\theta_{r}$ of the parameter Gibbs model. So, we propose the use of the Bootstrap methodology [3], which is successfully developed in different areas of applied statistic. It is to estimate the quantity $h_{D}\left(\theta_{r}, \theta_{1}\right)$ in (24) by $h_{D}\left(\theta_{1}, \theta_{1}^{*}\right)$, which is completely determined given the same initial observed realization $x$ of the Markov Random Field $X$. The value $\theta_{1}^{*}$ is the bootstrap estimation of the unknown value $\theta_{r}$ given the same initial observed configuration $x$ of $X$. because of this, we introduce the Bootstrap approximation of the adjusted interaction potential $\left(\mathrm{I}_{\mathrm{V}}\right)_{\mathrm{V}}$ as follow:

$\tilde{I}_{V}^{*}(x)=\left\{\begin{array}{cc}I_{V}\left(\theta_{1}, x\right)+\frac{|D|}{\left|\phi_{D}\right|} \cdot h_{D}\left(\theta_{1}, \theta_{1}^{*}\right), & \text { if } \operatorname{diam}(V) \leq \gamma \\ 0, & \text { otherwise }\end{array}\right.$

And we use the ergodic approximation of the MCMC $\left(X^{j}\right)_{j}$ with invariant distribution $\mu$ for the expected quantity as:

$$
\widehat{E}_{\mu}(g)=\frac{1}{N} \sum_{j=1}^{N} X^{j}
$$

However, to obtain an approximation of the quantity $h_{D}\left(\theta_{1}, \theta_{1}^{*}\right)$ in (26), we need more than two MCMC, due to the fact that we have as in (16) and (17):

$$
|D| . h_{D}\left(\theta_{1}, \theta_{1}^{*}\right)=E_{\theta_{1}}\left[\log \left(\frac{\pi_{\theta_{1}}^{D}(X)}{\pi_{\theta^{*}{ }_{1}}^{D}(X)}\right)\right]
$$

what requires a important computational time. Fortunately, we can write easily the following expression:

$$
\frac{z_{D}\left(\theta_{1}\right)}{z_{D}\left(\theta_{1}^{*}\right)}=E_{\theta_{1}^{*}}\left[\exp \left(\Delta H_{D}\right)\right]
$$

Where, 
b) Example of the approximation function $\tilde{h}_{D}\left(\theta_{1}, \theta_{1}^{*}\right)$ :

For the same Gibbs model above, we calculate the approximation function $\tilde{h}_{D}\left(\theta_{1}, \theta_{2}\right)$ for two various values $\theta_{1}$ et $\theta_{2}$, to examine the evolution of this function with regard to the difference between $\theta_{1}$ et $\theta_{2}$, using the MCMC simulated until different two states $(\mathrm{N}=600$ and $\mathrm{N}=1000)$. The case indicated by NA means that the denominator of $c(n)$ in (31) becomes a very high number.

TABLE I. THE VALUe OF $\tilde{h}_{D}\left(\theta_{1}, \theta_{2}\right)$ FOR DifFerent VALUes of $\theta_{1}$ ET $\theta_{2}$

\begin{tabular}{|l|l|l|l|l|}
\hline Value of $\theta_{1}$ & Value of $\theta_{2}$ & $\left|\theta_{1}-\theta_{2}\right|$ & $\mathrm{N}=600$ & $\mathrm{~N}=1000$ \\
\hline-10 & -9 & 1 & 0.0139 & 0.0116 \\
\hline-2 & -1.5 & 0.5 & 0.0077 & 0.0102 \\
\hline-1 & -0.23 & 0.77 & 0.0112 & 0.0086 \\
\hline-0.1 & -0.002 & 0.098 & 0.0039 & 0.0038 \\
\hline-0.3 & 0 & 0.3 & 0.0054 & 0.008 \\
\hline 0 & 0.5 & 0.5 & 0.0104 & 0.0106 \\
\hline 0.1 & 0.9 & 0.8 & 0.0166 & 0.0098 \\
\hline 0.3 & 1 & 0.7 & 0.0117 & 0.0110 \\
\hline 1 & 2 & 1 & 0.0162 & 0.0133 \\
\hline 2 & 3 & 1 & 0.0125 & 0.0106 \\
\hline 10 & 11 & 1 & 0.0120 & 0.0154 \\
\hline 10 & 12 & 2 & 0.01704 & 0.01593 \\
\hline 10 & 15 & 5 & NA & NA \\
\hline
\end{tabular}

This gives the shape of the two graphs of this function as follows:

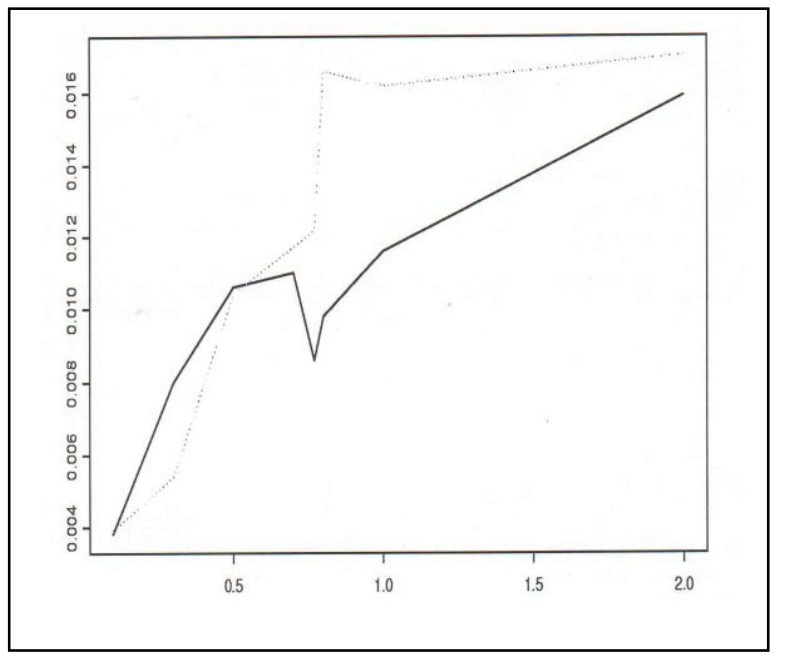

Fig. 2. The variation of the approximated function based on the difference between $\theta_{1}$ and $\theta_{2}$; the dotted-line curve for $\mathrm{N}=600$ stats of the $\mathrm{MCMCm}$ and the continuous-line curve for $\mathrm{N}=1000$

We can notice that the approximate function keeps the theoretical properties of the initial function, such as

$$
\tilde{h}_{D}\left(\theta_{1}, \theta_{2}\right) \cong 0 \text { if } \theta_{1} \cong \theta_{2}
$$

The main advantage of this approximation is in its easy use while keeping its strong properties as information criterion and adjusted term for the estimated potential. This assures the validity of the approximation of the Gibbs measure given above.

\section{REFERENCES}

[1] A. Benveniste, P. Metivier, Priouret, "Algorithmes adaptatifs et approximations stochastiques, théorie et application-techniques stochastiques". Masson, 1987.

[2] B.D. Ripley, "Spacial statistics". Wiley, New York. 1981

[3] B. Efron, "Bootstrap methods: Another look at Jackknife", Annals of Statistic, 7, 1-26. 1979.

[4] B. Efron, and Tibshirani, R., "An introduction to the Bootstrap". Monographs on statistics and applied Probability 57, Chapman and Hall. 1993.

[5] B. Gidds, "Parameter estimation for Gibbs distributions from fully observed data. In Markov Ranfom Fields: theory and applications". Cellapa, L. and Jain, A. (eds). 741-498. Academic Press, New York. 1993.

[6] B. Prum, "Processus sur un réseau et mesure de Gibbs; application". Techniques stochastiques, Masson. 1986.

[7] B. Simon, " A remarque on Dobrushin uniqueness theorem". Comm. Math-Phys. p.183. 1979

[8] C. Preston, "Random fields". In lect.nots in Math, vol. 534. Berlin, hedlber, New York, Springer. 1976.

[9] D Geman, and S. Geman, "Stochastic Relaxation, Gibbs Distribution and Baysian Restoration of Images". IEEE TPAM, vol-6 pp721-741. 1984.

[10] S. Singh, "On the asymptotic accuracy of Efron's Bootstrap". The Annals of Statistics vol. 9, N 6, 1187-1195. 1981.

[11] L. Dobrushin, "The description of a random field by means of conditional probabilities and it conditions of its regularity". Theory. Prob. Appl. XIII-2 pp 197-224. 1968.

[12] L. Tierney, " Markov Chains for exploring posterior distributions". Tech. Rep. 560, School of statistics, University of Minnesota. 1991.

[13] J. Besag, "Spatial interaction and the statistical analysis of lattice systems". J. Roy. Soc. Ser B, 36 192-236. 1974.

[14] P. Hall, "On the Bootstrap and Edgeworth expansion". New York, Spring. 1992.

[15] X. Guyon, "Champs aléatoires sur un réseau". Techniques Stochastiques, Masson. 1993.

[16] V. Ripley, "Modern Applied Statistics with Splus". Springer, New York, Berlin. 1996.

[17] W. K. Hastings, "Monte Carlo sampling methods using Markov Chain and their application". Biometrika, 57, 97-109. 1970.

[18] L. Younes, "Parametric inference for imperfectly observed Gibbsian fields. Prob. Theory related fields". 82 pp 625-645. 1989.

[19] L. Younes, "Maximum Likelihood Estimation for Gibbsian Fields". Lec. Notes-Monograph series, vol. 20. Spatial statistics and Imaging, pp 403426. 1991

[20] F. Comets, B. Gidas, "Parameter Estimation for Gibbs Distributions from Partially observed Data". The Annals of App. Probability, vol. 2, pp 142-170. 1992.

[21] H. Föllmer, J.L .Snell, “ An Inner Variational Principle for Markov Fields on Graph”, Gebiet, 39 pp 187-195. 1977. 